\title{
Probabilistic framework for opportunistic spectrum management in cognitive ad hoc networks
}

\author{
Ahmed Khattab*, Dmitri Perkins and Magdy A Bayoumi
}

\begin{abstract}
Existing distributed opportunistic spectrum management schemes do not consider the inability of today's cognitive transceivers to measure interference at the primary receivers. Consequently, optimizing the constrained cognitive radio network performance based only on the local interference measurements at the cognitive senders does not lead to truly optimal performance due to the existence of hidden (or exposed) primary senders. In this paper, we present a probabilistic framework for opportunistic spectrum management in cognitive ad hoc networks that optimizes the constrained cognitive user goodput while taking the unavoidable inaccuracy of spectrum sensing into account. The proposed framework (i) randomly explores individual spectrum bands as local interference measurements lead to inaccurate spectrum access decisions and (ii) adopts a non-greedy probabilistic spectrum access policy that prevents a single cognitive transmission from monopolizing an available spectral opportunity. In contrast to existing techniques, our approach allows multiple cognitive flows to fairly share the available opportunities without explicit inter-flow coordination. We analytically formulate the cognitive user performance optimization problem as a mixed-integer non-linear programming to derive the optimal parameter values. We use packet-level simulations to show that our approach achieves up to $138 \%$ higher goodput with significantly better fairness characteristics compared to greedy approaches.
\end{abstract}

Keywords: Cognitive radio networks, Opportunistic spectrum management, Medium access control

\section{Introduction}

The proliferation of the wireless communication industry has led to spectrum scarcity as the majority of spectrum has already been licensed. However, recent FCC measurements have shown that the licensed spectrum is underutilized for 15 to $85 \%$ of the time depending on the spatial location [1]. Thus, motivated cognitive radio networks (CRNs) have emerged as a solution for spectrum scarcity which explores the unutilized spatiotemporal spectral opportunities [2-4]. Several opportunistic spectrum sensing and management schemes have been proposed in the literature aiming at maximizing the CRN goodput while satisfying the constraints of the primary licensed networks (PRNs) [5-18]. However, such schemes do not take into account the practical limitations of CRNs.

\footnotetext{
* Correspondence: akhattab@ieee.org

The Center for Advanced Computer Studies (CACS), University of Louisiana at Lafayette, Lafayette, LA 70504, USA
}

\section{Springer}

On the one hand, cognitive radios are required to achieve sufficiently high sensitivity for a wide spectrum (e.g., multi-GHz) with high processing speed at low power consumption. However, existing hardware technologies do not meet such stringent requirements $[3,5,19]$. Furthermore, the finite sensing duration limits the spectrum sensing accuracy. Longer spectrum sensing windows are not necessarily useful since the environment is dynamic and the energy on a given channel is modulated both by the bursty traffic and the asynchronous initiation and termination of packet transmissions [5].

However, the most important factor that limits the accuracy of spectrum sensing is that most of the existing techniques adopt some form of the traditional listenbefore-talk strategy to detect the activities of the primary transmitters. Currently, there does not exist any practical way that allows cognitive nodes, also called secondary users (SUs), to measure the interference at 
nearby primary network receivers [3-5] since primary users (PUs) are passive and do not interact or share information with SUs. ${ }^{a}$ Therefore, interference measurements based on local observations at SUs are inaccurate. Such erroneous spectrum measurements cause the SUs to mistakenly infer spectral opportunities or miss spectral opportunities as is the case in the scenarios depicted in Figure 1a, b, respectively.

On the other hand, the coordination between multiple secondary users is a major challenge in distributed multiuser cognitive radio networks. If legacy MAC protocols designed for traditional networks were to be used in CRNs, all of the secondary users that infer a spectral opportunity will greedily attempt to exploit the sensed opportunity. Recall that legacy MACs often adopt greedy strategies that try to best utilize a spectrum access (e.g., by using the highest transmission rate or choosing the best channel). Such greedy approaches deteriorate the goodput performance of a CRN as the number of SUs increases due to increased blocking probability [3,4]. Furthermore, such greedy MACs are known to suffer from unfairness problems that can cause some secondary sender-receiver pairs to dominate other pairs. Several distributed cooperative MAC approaches have been recently developed for CRNs

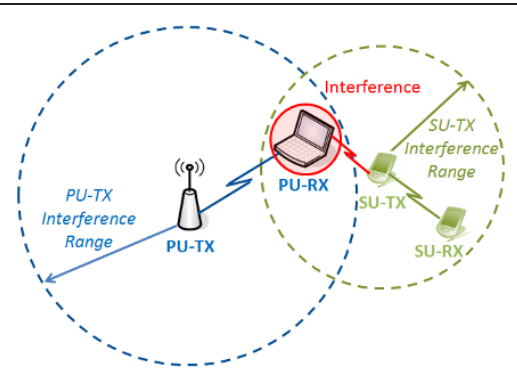

(a) Hidden primary sender scenario.

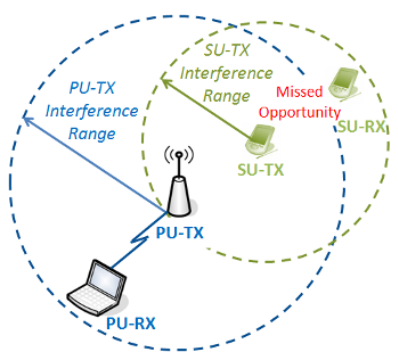

(b) Exposed primary sender scenario.

Figure 1 Example problematic scenarios. The primary network transmission will be intercepted by the secondary transmission initiated due to a miss-predicted spectral opportunity as shown in Figure 1a. Meanwhile, the secondary user misses a spectral opportunity because of the misleading interference measurement as depicted in Figure 1b. a Hidden primary sender scenario; b Exposed primary sender scenario.
$[12,14,16]$. However, such distributed schemes rely on the explicit coordination between different flows which is a main challenge in CRNs as it requires gathering and distributing spectrum information across the CRN and/ or synchronizing the activities of different flows. Such explicit inter-flow coordination further deteriorates the CRN goodput and heavily depends on the common control channel (also used for the coordination between a sender and its respective receiver) and causes it to be the bottleneck of a CRN and the single point of failure for the entire system $[3,4]$.

\subsection{Our contributions}

Our objective is to realize a practical spectrum management scheme for cognitive radio networks that (i) counters the unavoidable inaccuracies in spectrum measurements and their consequent negative impact on the CRN and PRNs performance and (ii) allows secondary users to fairly share the spectral opportunities without explicit inter-flow coordination. The proposed scheme relaxes the hardware requirements of the cognitive transceivers. We address the following two open questions assuming a decentralized asynchronous ad hoc CRN. First, given that a secondary sender does not apriori know the impact of its transmission on nearby primary receivers, how aggressive/conservative a secondary sender should/should not be to alleviate spectral miss-predictions and missed opportunities. Second, how non-greedy spectrum access can allow multiple secondary users to share spectral opportunities without explicit information sharing. Our contributions are as follows.

First, we propose the rate-adaptive probabilistic (RAP) spectrum management framework and its medium access control protocol realization (RAP-MAC). The main ideas behind our framework are as follows: (i) any spectrum band can be explored with a certain probability-even if the measured interference level is high-since the local interference measurements at the CRN senders do not infer the interference at nearby primary receivers; (ii) a CRN transmission does not greedily exploit a spectral opportunity. Instead, a CRN transmission probabilistically switches between the maximum permissible transmission power/rate and lower powers/rates. Thereby, RAP-MAC probabilistically reduces the potential harm to nearby primary receivers and leaves a spectral margin for other CRN flows to transmit. In multiuser ad hoc networks, RAP-MAC adaptively makes different CRN flows share the spectral opportunities without explicit inter-flow coordination. In contrast, hypothetically optimal spectrum management schemes greedily transmit only over the channel(s) with the least primary interference at the maximum permissible power/rate and rely on an explicit inter-flow coordination mechanism. 
Second, we analytically formulate the constrained CRN optimization problem according to the RAP framework in order to compute the optimal probabilities of transmission and the used rates and powers. In our formulation, we consider another practical limitation of CRN hardware that is only a finite set of transmission powers/rates is available. This limitation causes our optimization problem to be a mixed-integer non-linear programming which complexity is NP-complete. We present an exhaustive study of the impact of various factors on the optimal RAP-MAC parameter values. More specifically, we investigate the impact of the primary networks' outage constraints and user activity factors on the optimal probabilities of the RAP-MAC protocol as well as the achievable cognitive user goodput.

Finally, we use packet-level simulations to demonstrate that RAP-MAC probabilistic spectrum management achieves up to $138 \%$ higher goodput compared to greedy spectrum management depending on the CRN traffic demand. This superior performance is attributed to the RAP-MAC probabilistic sensing and transmission policies, which explores more spectral opportunities and leads to fewer transmission failures compared to deterministic and hypothetically optimal spectrum management. Furthermore, RAP-MAC results in different CRN flows fairly sharing the available opportunities without explicit inter-flow coordination. Meanwhile, greedy spectrum management results in $47 \%$ of the flows receiving less than $10 \%$ of the average goodput. Our approach satisfies the primary network performance constraints despite the use of cognitive transceivers with narrowband sensing capability compared to hypothetically optimal spectrum management that assumes wideband cognitive transceivers.

The remainder of the paper is organized as follows. In Section 2, we define the system model. We propose the RAP framework and protocol in Section 3 then compute its optimal parameter values in Section 4. In Section 5, we exhaustively study the performance of RAP-MAC via simulations. We review the related literature in Section 6 and conclude in Section 7.

\section{System model}

\section{Primary Network Model}

We consider a wireless spectrum consisting of $N$ nonoverlapping channels. We assume $N$ distinct primary radio networks (PRNs) licensed to operate in these $N$ channels. ${ }^{\mathbf{b}}$ All of the $N$ PRNs are geographically collocated. The maximum transmission power of the $i$ th PRN is $P_{\mathrm{PU}}^{(i)}$. The PRN user distributions are modeled as homogeneous Poisson random processes with parameters $\rho_{i}$ representing the user density of the $i$ th PRN. A primary user (PU) in the $i$ th PRN is modeled as an
ON/OFF source with activity factor $\alpha_{i}$ defined as the fraction of time the user in ON. PRNs are non-intrusive and operate as they are the sole users of their licensed spectrum. PUs do not provide any type of cooperation with the underlaying secondary network. However, each PRN defines the maximum permissible interference margin from the secondary network. We denote such a power mask of the $i$ th PRN (and consequently the $i$ th channel) as $P_{\text {mask }}^{(i)}$. We adopt a statistical model that ensures that the cumulative interference from the secondary user activities does not exceed $P_{\text {mask }}^{(i)}$ with probability $\beta$, thereby providing a mask stochastic guarantee on the performance of PUs.

\section{Secondary Network Model}

We consider a single ad hoc secondary cognitive radio network (CRN) that is geographically collocated with the $N$ PRNs. Transmissions within different PRNs and the CRN can start at any arbitrary time instant (i.e., we do not assume a time-slotted system). The unlicensed users of the CRN can opportunistically access any of the $N$ non-overlapping channels, one channel at a given time. A secondary user (SU) is equipped with a single cognitive radio transceiver that can be tuned to transmit over any of the $N$ channels. We assume the transceiver has a narrowband sensing capability. That is, a SU transceiver can only sense a single channel at a time. While not optimal compared to wideband sensing, narrowband spectrum sensing relaxes the hardware complexity and the power consumption of SU terminals (especially for low-cost battery-powered devices). SUs are of lower priority with respect to spectrum access compared to the spectrum's licensed PUs. The secondary user density is $\rho_{\mathrm{SU}}$. We consider a multiuser CRN environment in which one or more SUs can transmit over a given channel once an access opportunity is inferred (i.e., the sensed cumulative interference power on the $i$ th channel is less than $\left.P_{\text {mask }}^{(i)}\right)$. We denote the transmission power of the $j$ th SU over the $i$ th channel as $P_{S U_{j}}^{(i)}$ and the corresponding transmission rate as $r_{S U_{j}}^{(i)}$. Both $P_{S U_{j}}^{(i)}$ and $r_{S U_{j}}^{(i)}$ are fixed throughout a packet transmission. A SU can choose its rate from a finite set of available rates $R_{1}<R_{2}<\ldots$ $<R_{m}$. Each rate $R_{i}$ has a corresponding distinct transmission power $P_{1}<P_{2}<\ldots<P_{m}$. The powers $P_{i}$ s are such that the transmission range is fixed irrespective of the used rate. Thus, the following relationship holds for any pair of rates

$$
\frac{P_{i}}{P_{j}}=\frac{2^{R_{i}}-1}{2^{R_{j}}-1}, \quad \forall i \neq j
$$


due to the logarithmic relationship between the rate and power regardless of the used physical layer scheme [20]. A secondary sender-receiver pair coordinates its spectrum selection and transmission policy using a dedicated common control channel in the unlicensed band. Unlike prior work, the common control channel is not used for any sort of inter-flow coordination.

\section{Rate-adaptive probabilistic approach for opportunistic spectrum access}

In this section, we propose the rate-adaptive probabilistic (RAP) framework for spectrum sensing and management and its protocol implementation RAP-MAC.

\subsection{RAP framework}

The proposed RAP framework has two main components: The randomized spectrum selection component that addresses the spectral sensing problems, combined with the rate-adaptive probabilistic transmission policy which probabilistically: (i) allows secondary senders to better explore spectral opportunities regardless of the inaccuracy of spectrum sensing and (ii) enables multiple secondary flows to share the available opportunities in a distributed manner without explicit inter-flow coordination.

\subsubsection{Coordinated random spectrum selection}

As we explained earlier, secondary senders are unable to apriori assess the impact of their transmissions on nearby primary receivers based on the PU interference measurements. Consequently, secondary transmitters make wrong spectrum access decisions due to missjudged spectral opportunities. Our spectrum sensing scheme relaxes the constraints on the spectrum sensing hardware and counters potential inaccuracies via the following two ideas.

Randomized Spectrum Selection A secondary transmitter (SU-TX) randomly selects a spectrum to probe for an upcoming transmission (if there does not exist a preferred spectrum that recently carried out a successful transmission). Due to the inability of a secondary sender to accurately assess the impact of its transmission on ongoing transmissions, a secondary sender can choose any spectrum with equal probability for an upcoming transmission. Prior work used randomized spectrum sensing to spread multiple SUs over different spectrum bands $[7,8]$. However, such schemes require the exact apriori knowledge of the statistics of the activities of primary users and the number of competing SUs in order to compute the probability of sensing a particular spectrum band. In contrast, we use randomization to relax the cognitive radio requirements and alleviate the need for wideband sensing given the inherent inaccuracy of spectrum sensing.
Coordinated Sender-Receiver Sensing In ad hoc environments in which nodes are exposed to different parts of the network, the interference at the sender and receiver of a SU flow is typically different. Therefore, the spectrum access decision must be based on the view of the spectrum at both endpoints of the transmission (not only on the sender's view of the spectrum as the case with traditional listen-before-talk MAC protocols). Hence, the RAP framework has the secondary receiver (SU-RX) also measuring the interference over the secondary-sender-selected spectrum. Given the interference measurements of the selected spectrum at both the SUTX and SU-RX, four scenarios arise. In the first scenario, both measurements indicate low interference (i.e., the cumulative interference is below the power mask). We refer to such scenario as a clear spectral opportunity. The second scenario is when the SU-TX is experiencing strong interference (i.e., the cumulative interference exceeds the power mask) and the SU-RX is experiencing low interference. We refer to such scenario as an unclear spectral opportunity. The other two scenarios are when the spectrum measurement at the SURX indicates high interference levels. In such scenarios, the SU-RX will not be able to correctly receive the data over the selected spectrum. The RAP framework avoids unnecessary usage of such a spectrum band by having the SU-TX randomly selecting a new spectrum.

\subsubsection{Rate-adaptive probabilistic transmission}

Even with the spectrum measurements at both the SUTX and SU-RX, the decision of whether or not to use the sensed spectrum cannot be accurate. We propose the following probabilistic spectrum access scheme which is: (i) conservative and non-greedy in exploiting clear spectral opportunities, and hence, it probabilistically reduces PRN outages due to spectral miss-predictions while allowing multiple secondary flows to exploit a given spectral opportunity; and (ii) probabilistically nonconservative in exploiting unclear spectral opportunities in order to reduce CRN goodput degradation due to spectral missed opportunities.

Clear Spectral Opportunity In clear spectral opportunity scenarios, the RAP framework exploits the senderselected spectrum at the maximum permissible power/ rate only with a certain probability $p$ (since a SU-TX does not know for sure if its transmission will interfere with any ongoing primary receptions or not). Besides, such a non-greedy medium access approach does not allow a SU-TX to fully utilize the available capacity of a given spectral opportunity since the SU-TX does not transmit at the highest possible power and rate. Instead, a SU-TX probabilistically leaves a capacity margin by using a lower power/rate with probability $(1-p)$. Hence, if there exists a neighboring $\mathrm{SU}$ transmission, it can 
exploit such a capacity margin to announce its presence. Consequently, different $\mathrm{SU}$ transmissions adjust their powers and rates to share such an opportunity.

While potentially degrading the CRN goodput, the use of low power/rate transmission reduces the probability of intercepting ongoing unidentified PRN transmissions since the lower the rate, the lower its power. Starting from the minimum values, a SU-TX increases the rate and power used with probability $(1-p)$ to the next higher values upon a successful transmission until either the second highest values are reached or a transmission failure occurs. The purpose of the former condition is to not sacrifice the goodput of the CRN if there does not exist any nearby SU transmissions by gradually shrinking the unutilized capacity margin. Meanwhile, if a nearby secondary transmission decides to explore the same spectrum, it will cause the high rate transmission to fail. In this case, our scheme will have a SU-TX reverting to the lowest power/rate for future transmissions. Low power/rate communication scheme is more robust to interference that cannot be explicitly nulled out [20]. It was shown that multiple low power and low rate transmissions successfully coexist without explicit interference suppression [21].

Unclear Spectral Opportunity In unclear spectral opportunity scenarios, the RAP framework allows a SUTX to probabilistically transmit over the sender-selected spectrum with a certain probability $q$ (since not using the spectrum at all can lead to unnecessarily missing the opportunity). Otherwise, the SU-TX will search for another spectrum to use with probability $(1-q)$. Here, the SU-TX only uses the minimum power/rate due to their robustness to interference and their weak impact on ongoing transmissions. The SU-TX does not gradually increase its rate and power any further as it still cannot exactly assess its impact on the reception of nearby transmissions. In Section 4, we calculate the optimal values of $p$ and $q$ that maximize the CRN goodput while satisfying the PRN performance grantees.

\subsection{RAP-MAC protocol}

Algorithm 1 depicts RAP-MAC: the protocol implementation of the RAP framework. RAP-MAC is a four-way handshake protocol. A Spectrum Request (SR) and a Spectrum Grant (SG) message exchange precedes every packet transmission to communicate the spectrum selection and interference measurements of the SU-TX and SU-RX, respectively. The SR and SG packets are transmitted over the common control channel only to coordinate between a secondary sender and its respective receiver and not for inter-flow coordination as the case with the existing related literature $[12,14,16,22]$. If the SU-TX correctly receives the SG packet, it transmits a data packet over the selected spectrum at the rate and power probabilistically chosen as described above. If the SU-TX receives the ACK packet before the timeout timer expires, it declares the used spectrum as its favorite spectrum for upcoming transmissions if the used rate is greater than $R_{1}$. Otherwise, the SU-TX sets its favorite spectrum to null.

\section{RAP-MAC performance optimization with statistical PRN guarantees}

In this section, we analytically derive the optimal values of the parameters of the RAP-MAC protocol. More specifically, we find the values of the probabilities $p$ and $q$ along with the maximum secondary transmission rates and powers that maximize the average rate of a secondary user while providing statistical guarantees for the performance of PRNs. Typically, the performance of a PRN is defined in terms of its outage probability [3-8,12,14,16-18]. For each primary user $j$ in the $i$ th PRN, the outage probability $P_{\text {out }}^{(i)}\left(P U_{j}\right)$ is bounded by $\beta$. The constrained CRN optimization problem is formulated as follows

$$
\begin{array}{ll}
\text { maximize } & \sum_{i=1}^{N} \frac{1}{N} \cdot r_{\mathrm{SU}}^{(i)} \\
\text { subject to } & p_{\text {out }}^{(i)}(P U j) \leq \beta \quad \forall i=1,2, \ldots, N ; j=1,2, \ldots
\end{array}
$$

We next formulate this generic problem in terms of the RAP-MAC framework to find the optimal values of its parameters. For the ease of presentation, Table 1 lists the used notations.

\subsection{RAP-MAC achievable flow rate}

First, we compute the average rate a SU can achieve over the $i$ th channel, $r_{\mathrm{Su}}^{(i)}$, using the possible transmission rates and their corresponding RAP-MAC probabilities. Given the interference measurements at the sender and the receiver, there exists two possible cases that allow the secondary sender-receiver pair to use the randomly selected channel. The first case is the clear spectrum case in which the interference measurements at both endpoints are below the interference threshold of this particular channel. In the second case of unclear spectrum, only the interference measured at the secondary receiver is below the threshold. Due to the independence of the interference measurements at the sender and its receiver, the probabilities of the two cases are $\left(\operatorname{Pr}\left[P_{\text {int }}^{(i)} \leq P_{\text {mask }}^{(i)}\right]\right)^{2}$ and $\operatorname{Pr}\left[P_{\text {int }}^{(i)} \leq P_{\text {mask }}^{(i)}\right]\left(1-\operatorname{Pr}\left[P_{\text {int }}^{(i)} \leq P_{\text {mask }}^{(i)}\right]\right)$, respectively, where $P_{\text {int }}$ is the random variable representing the interference experienced at a SU terminal over the $i$ th spectrum band. The probability distribution of $P_{\text {int }}^{(i)}$ was approximated in [16] by a lognormal distribution with mean and variance given by 
Table 1 List of used notations.

\begin{tabular}{ll}
\hline Parameter & Definition \\
\hline$n$ & Propagation path loss exponent \\
$d_{c}$ & Distance beyond which the interference is negligible (i.e., below the receiver sensitivity) \\
$\lambda^{(i)}$ & Operating wavelength of the ith PRN \\
$G_{T_{i}}^{(i)}$ & Transmit antenna gain of the ith PRN \\
$G_{R}^{(i)}$ & Receive antenna gain of the ith PRN \\
$d_{0}^{(i)}$ & Close-in distance of the ith PRN \\
$P_{o}^{(i)}$ & Reference power at the close-in distance of the ith PRN $P_{o}^{(i)}=\frac{P_{\mathrm{PU}}^{(i)} G_{T}^{(i)} G_{R}^{(i)} \lambda^{(i)^{2}}}{4 \pi d_{o}^{(i)^{2}}}$ \\
$\alpha_{i}$ & Activity factor of the ith PRN \\
$\rho_{i}$ & User density of the ith PRN \\
$\rho_{\mathrm{SU}}$ & User density of the CRN \\
$P_{\operatorname{ma}}^{(i)}$ & Power mask of the ith PRN \\
$P_{\max }^{(i)}$ & Maximum SU power to be used over the ith spectrum \\
$R_{\mathrm{max}}^{(i)}$ & Maximum SU rate to be used over the ith spectrum \\
$R_{\max -1}^{(i)}$ & Second highest SU rate to be used over the ith spectrum \\
$R_{1}$ & Minimum SU rate \\
$\operatorname{erfc}(\cdot)$ & Complementary error function [20] \\
\hline
\end{tabular}

Algorithm 1 Pseudocode of the RAP-MAC protocol

\section{SU-TX Spectrum Request \\ if current_spectrum $=0$ then}

choose $i \in\{1, \ldots, N\}$ with probability $1 / N$

current_spectrum $=i$

\section{end if}

$P_{\text {int }}^{t x}=$ spectrum_measure(current_spectrum)

Send(SR(current_spectrum, $\left.\left.P_{\text {int }}^{t x}\right)\right)$

\section{SU-RX Spectrum Grant} receive(SR(current_spectrum, $\left.\left.P_{i n t}^{t x}\right)\right)$

$P_{\text {int }}^{r x}=$ spectrum_measure(current_spectrum)

if $\left(P_{\text {int }}^{t x}<P_{\text {mask }}^{(i)}\right)$ and $\left(P_{\text {int }}^{r x}<P_{\text {mask }}^{(i)}\right)$ then

clear_spectrum $=1$

send (SG $\left(R_{\max }^{(i)}\right.$, clear_spectrum))

else if $\left(P_{i n t}^{t x} \geq P_{\text {mask }}^{(i)}\right)$ and $\left(P_{i n t}^{r x}<P_{\text {mask }}^{(i)}\right)$ then

clear_spectrum $=0$

$\operatorname{send}\left(\mathrm{SG}\left(R_{1}\right.\right.$, clear_spectrum $\left.)\right)$

\section{end if}

\section{SU-TX Data Packet Transmission}

receive(SG(r, clear_spectrum))

if clear_spectrum and Single_SU then

rate $=R_{\max }^{(i)}$ with probability $p$

rate $=R_{\min }$ with probability $1-p$

send(DATA)

else if clear_spectrum and not Single_SU then

rate $=R_{1}$

send(DATA)

else

rate $=R_{1}$

send(DATA) with probability $q$

end if

SU-TX Receiving Acknowledgement if receive(ACK) and $R_{\min }<R_{\max -1}^{(i)}$ then

Single_SU $=1$

increase $\left(R_{\text {min }}\right)$

else

current_spectrum $=0$

Single_SU $=0$

$R_{\text {min }}=R_{1}$

end if

$E\left[P_{\text {int }}^{(i)}\right]= \begin{cases}2 \pi \alpha_{i} \rho_{i} P_{o}^{(i)} d_{o}^{(i)^{2}} e^{-\pi \alpha_{i} \rho_{i} i_{o}^{(i)^{2}}} \ln \frac{d_{c}}{d_{o}^{(i)}}, n=2 \\ \frac{2 \pi \alpha_{i} \rho_{i} \rho_{o}^{(i)} d_{o}^{(i)^{2}}}{n-2} \mathrm{e}^{-\pi \alpha_{i} \rho_{i} d_{o}^{(i))^{2}},}, \quad n>2\end{cases}$

and

$$
\operatorname{Var}\left[P_{\text {int }}^{(i)}\right]=\frac{\pi \alpha_{i} \rho_{i}}{n-1}\left[2 P_{0}^{(i)} d_{o}^{(i)^{2}} e^{-\pi \alpha_{i} \rho_{i} d_{o}^{(i)^{2}}}\right]^{2}, \quad n \geq 2
$$

respectively. Given the statistics of the distribution of $P_{\text {int }}^{(i)}$, the probabilities of the clear and unclear spectrum are given by

$$
p_{\text {clear }}=\left[\frac{1}{2} \operatorname{erfc}\left(-\frac{\ln P_{\text {mask }}^{(i)}-\mu_{P_{\text {int }}^{(i)}}}{\sqrt{2 \sigma_{P_{\text {int }}^{(i) ~}}^{2}}}\right)\right]^{2}
$$

and

$$
\begin{aligned}
p_{\text {unclear }}=\frac{1}{2} \operatorname{erfc} & \left(-\frac{\ln P_{\text {mask }}^{(i)}-\mu_{P_{\text {int }}^{(i)}}}{\sqrt{2 \sigma_{P_{\text {int }}^{(i)}}^{2}}}\right) \\
\times & {\left[1-\frac{1}{2} \operatorname{erfc}\left(-\frac{\ln P_{\text {mask }}^{(i)}-\mu_{P_{\text {int }}^{(i)}}}{\sqrt{2 \sigma_{P_{\text {int }}^{(i)}}^{2}}}\right)\right] }
\end{aligned}
$$


respectively, where

$$
\begin{aligned}
& \mu_{P_{\text {int }}^{(i)}}=\ln \left(E\left[P_{\text {int }}^{(i)}\right]\right)-\frac{1}{2} \ln \left(1+\frac{\operatorname{Var}\left[P_{\text {int }}^{(i)}\right]}{E\left[P_{\text {int }}^{(i)}\right]^{2}}\right) \\
& \sigma_{P_{\text {int }}^{(i)}}^{2}=\ln \left(1+\frac{\operatorname{Var}\left[P_{\text {int }}^{(i)}\right]}{E\left[P_{\text {int }}^{(i)}\right]^{2}}\right)
\end{aligned}
$$

According to RAP-MAC, the rate of a sender-receiver pair is $q R_{1}$ in the unclear spectral opportunity case. We next calculate the average secondary flow rate whenever the spectrum is measured to be clear. The flow rate given no other secondary senders is in the vicinity of the tagged secondary receiver and using the selected channel is $p R_{\max }^{(i)}+(1-p) R_{\max -1}^{(i)}{ }^{\mathbf{c}}$ Meanwhile, the flow rate is $R_{1}$ if there exists at least one more SU transmitting on the selected spectrum in the vicinity of the tagged secondary receiver. The probability of having at least one more secondary sender over the selected channel in the receiver's vicinity is the probability of having $k \geq 2$ secondary senders and one minus the probability of only the tagged sender selecting the $i$ th channel while the remaining $k-1$ senders select different channels. Since the locations of the secondary users are modeled as a homogeneous Poisson process, the probability of the number of potential senders within a disk area $A_{c}=\pi d_{c}^{2}$ is equal to $k$ is given by

$$
\operatorname{Pr}[K=k]=\frac{e^{-\rho_{\mathrm{SU}} A_{c}}\left(\rho_{\mathrm{SU}} A_{c}\right)^{k}}{k !}, \quad k=0,1,2, \ldots
$$

Hence, the probability of multiple concurrent secondary transmissions over the $i$ th channel, $p_{\mathrm{MSU}}$, is given by

$$
\begin{aligned}
p_{\mathrm{MSU}} & =\sum_{k=2}^{\infty} \frac{\mathrm{e}^{-\rho_{\mathrm{SU}} A_{c}}\left(\rho_{\mathrm{SU}} A_{c}\right)^{k}}{k !} \cdot\left(1-\frac{1}{N}\left(\frac{N-1}{N}\right)^{k-1}\right) \\
& =1-\mathrm{e}^{-\rho_{\mathrm{SU}} A_{c}}-\frac{\mathrm{e}^{-\frac{\rho_{\mathrm{SU}} A_{c}}{N}}}{N-1}+\frac{\mathrm{e}^{-\rho_{\mathrm{SU}} A_{c}}}{N-1}
\end{aligned}
$$

where $\left(1-\frac{1}{N}\left(\frac{N-1}{N}\right)^{k-1}\right)$ is the probability that at least one other SU sender selects the same channel. Similarly, the probability of no other concurrent secondary transmission, $p_{\mathrm{SSU}}$, is computed using the probability of the two events of either no other nearby sender exists (i.e., the probability of $k<2$ ) or none of the $k \geq 2$ nearby senders selects the same channel as the tagged sender as

$$
\begin{aligned}
p_{\mathrm{SSU}}= & \mathrm{e}^{-\rho_{\mathrm{SU}} A_{c}}\left(1+\rho_{\mathrm{SU}} A_{c}\right) \\
& +\sum_{k=2}^{\infty} \frac{\mathrm{e}^{-\rho_{\mathrm{SU}} A_{c}}\left(\rho_{\mathrm{SU}} A_{c}\right)^{k}}{k !} \cdot \frac{1}{N}\left(\frac{N-1}{N}\right)^{k-1} \\
= & e^{-\rho_{\mathrm{SU}} A_{c}}+\frac{\mathrm{e}^{-\frac{\rho_{\mathrm{SU}} A_{c}}{N}}}{N-1}-\frac{\mathrm{e}^{-\rho_{\mathrm{SU}} A_{c}}}{N-1}
\end{aligned}
$$

Using the probabilities of clear and unclear spectrum given by (5) and (6) and the multiple and single SU probabilities given by (10) and (11), the average rate of a $\mathrm{SU}$ is written as

$$
\begin{aligned}
r_{\mathrm{SU}}^{(i)}=[( & \left.p R_{\max }^{(i)}+(1-p) R_{\max -1}^{(i)}\right) p_{\mathrm{SSU}} \\
& \left.+R_{1} p_{\mathrm{MSU}}\right] p_{\mathrm{cl}_{\mathrm{ear}}}+q R_{1} P_{\text {unclear }}
\end{aligned}
$$

\subsection{Statistical PRN outage constraints}

Next, we formally define the statistical constraints on the outage probability given in (2) in terms of $p, q$, and the maximum secondary user transmission power over different spectrum bands. For a given secondary transmitter, all of the surrounding primary receivers must successfully receive their intended data with probability $1-\beta$. This constraint is satisfied if and only if it is satisfied at the primary receiver that is closely located with respect to the secondary sender. Let's denote the minimum distance between a secondary sender and the closest primary receiver by $\mathcal{D}_{\text {min. }}$. We define the outage probability $p_{\text {out }}^{(i)}$ at the $i$ th PRN receiver at distance $\mathcal{D}$ as follows

$$
\begin{gathered}
p_{\text {out }}^{(i)}=\operatorname{Pr}[\text { SU - TX }]\left(\operatorname{Pr}\left[\text { outage } \mid \mathcal{D}<\mathcal{D}_{\min }^{(i)}\right] \operatorname{Pr}\left[\mathcal{D}<\mathcal{D}_{\text {min }}^{(i)}\right]\right. \\
\left.+\operatorname{Pr}\left[\text { outage } \mid \mathcal{D} \geq \mathcal{D}_{\min }^{(i)}\right] \operatorname{Pr}\left[\mathcal{D} \geq \mathcal{D}_{\text {min }}^{(i)}\right]\right)
\end{gathered}
$$

where $\operatorname{Pr}[\mathrm{SU}-\mathrm{TX}]$ is either $p$ or $q$ depending on the interference measurements at the secondary flow endpoints, and $\mathcal{D}_{\text {min }}^{(i)}$ is a random variable that models the minimum distance between a secondary sender and a primary receiver in the $i$ th PRN. The probabilities of the two events $\mathcal{D}<\mathcal{D}_{\text {min }}^{(i)}$ and $\mathcal{D} \geq \mathcal{D}_{\text {min }}^{(i)}$ are computed using the cumulative distribution of the minimum distance between a SU-TX studied in [16,23]. According to our system model, the cumulative distribution function of $\mathcal{D}_{\text {min }}^{(i)}$ is given by

$$
F_{\mathcal{D}_{\min }^{(i)}}(d)=\operatorname{Pr}\left[\mathcal{D}_{\min }^{(i)}<d\right]=1-\mathrm{e}^{-\pi \alpha_{i} \rho_{i} d^{2}}
$$

Let's define $D_{\min }^{(i) *}$ to be the minimum distance below which the probability of outage is unity, that is, $\operatorname{Pr}\left[\right.$ outage $\left.\mid \mathcal{D}<\mathcal{D}_{\min }^{(i) *}\right] \triangleq 1$. According to $(14), D_{\min }^{(i)}$ is at least $D_{\text {min }}^{(i) *}$ with probability $p_{\mathcal{D}_{\min }^{*}}^{*}=1-\operatorname{Pr}\left[\mathcal{D}_{\min }<D_{\min }^{(i) *}\right]$. Substituting in (14), we get

$$
\mathcal{D}_{\min }^{(i) *}=\sqrt{\frac{-\ln \left(p_{\mathcal{D}_{\min }^{*}}\right)}{\pi \alpha_{i} \rho_{i}}}
$$

Note that, $p_{\mathcal{D}_{\text {min }}^{*}}$ determines how much $D_{\min }^{(i)}$ is close to $D_{\text {min }}^{(i) *}$. Give that $\operatorname{Pr}\left[\right.$ outage $\left.\mid \mathcal{D}<\mathcal{D}_{\text {min }}^{(i) *}\right] \triangleq 1$, and let $\gamma^{(i)}$ denote the conditional outage probability $\operatorname{Pr}$ [outage $\mid \mathcal{D}<\mathcal{D}_{\min }^{(i) *}$, the outage probability given by 
(13) can be rewritten as

$$
p_{\text {out }}^{(i)}=\operatorname{Pr}[\mathrm{SU}-\mathrm{TX}]\left(\left(1-p_{\mathcal{D}_{\text {min }}^{*}}\right)+\gamma^{(i)} p_{\mathcal{D}_{\text {min }}^{*}}\right)
$$

Hence, the $p_{\text {out }}^{(i)} \leq \beta$ constraints in (2) are equivalent to

$$
\gamma^{(i)} \leq 1-\frac{1-\frac{\beta}{\operatorname{Pr}[\mathrm{SU}-\mathrm{TX}]}}{p_{\mathcal{D}_{\min }^{*}}}
$$

Since $\gamma^{(i)}$ cannot be negative, $\operatorname{Pr}[\mathrm{SU}-\mathrm{TX}]$ must be no less than $\beta$ and the following constraint must be satisfied

$$
\operatorname{Pr}[\mathrm{SU}-\mathrm{TX}] \leq \frac{\beta}{1-p_{\mathcal{D}_{\text {min }}^{*}}}
$$

Finally, we relate the outage probability of the $i$ th channel to the $i$ th PRN power mask and the maximum power a SU can use over that channel. In order to preserve the required bounds on $p_{\text {out }}^{(i)}\left(P U_{j}\right)$, the following condition at every primary receiver $j$ should be satisfied with probability $\left(1-\gamma^{(i)}\right) \operatorname{Pr}[$ SU-TX] due to every secondary transmission

$$
P_{\text {int }, j}^{(i)}+g_{\mathcal{D}_{\text {min }}^{*}}^{(i)} P_{\text {SU }}^{(i)} \leq P_{\text {mask }}^{(i)}
$$

where $P_{\text {int, }}^{(i)}$ is the interference power at the $j$ th primary receiver due to other potential interfering activities, and $g_{\mathcal{D}_{\text {min }}^{*}}^{(i)}=\frac{G_{T}^{(i)} G_{R}^{(i)} \lambda^{(i)^{2}}}{(4 \pi)^{2}\left(\mathcal{D}_{\text {min }}^{(i) *}\right)^{n}}$ is the channel gain between the nearest secondary sender and the $j$ th primary receiver. Since RAP-MAC allows a secondary sender to use different transmission powers with certain probabilities, it is sufficient that the maximum permissible power $P_{\max }^{(i)}$ which is used with probability $\operatorname{Pr}[\mathrm{SU}-\mathrm{TX}]=p$ satisfies the condition in (19). ${ }^{\mathbf{d}}$ In order to satisfy (19) with probability $\left(1-\gamma^{(i)}\right) p$, we compute the $[(1-\gamma) p]$-quantile of $P_{\text {int, }}^{(i)}$ and substitute in (19). According to [16], $P_{\text {int, }}^{(i)}$ has a lognormal distribution, and hence, its $\left[\left(1-\gamma^{(i)}\right) p\right]$-quantile $P_{(1-\gamma) p}^{(i)}$ is calculated as

$$
P_{(1-\gamma) p}^{(i)}=\exp \left(-\sqrt{2 \operatorname{Var}\left[P_{\text {int }}^{(i)}\right]} \operatorname{erfc}^{-1}\left(2\left(1-\gamma^{(i)}\right) p\right)\right)
$$

Substituting with (20) in (19), we get the following constraint on the maximum transmission power of a secondary user over the $i$ th channel

$$
P_{\max }^{(i)} \leq \frac{P_{\text {mask }}^{(i)}-P_{(1-\gamma) p}^{(i)}}{g_{\mathcal{D}_{\min }^{*}}^{(i)}}
$$

\subsection{RAP-MAC parameter optimization}

Given $r_{\text {SU }}^{(i)}$ formulated in terms of $p$ and $q$ as in (12), the original optimization problem given in (2) can be restated in terms of the RAP-MAC parameters as follows

$$
\begin{gathered}
\text { maximize } \sum_{i=1}^{N} \frac{1}{N} \cdot r_{\mathrm{SU}}^{(i)} \\
\text { subject to } P_{\max }^{(i)} \leq \frac{P_{\text {mask }}^{(i)}-P_{(1-\gamma) p}^{(i)}}{g_{\mathcal{D}_{\min }^{*}}^{(i)}} \quad \forall i=1,2, \ldots, N \\
\beta \leq p \leq \frac{\beta}{1-p_{\mathcal{D}_{\text {min }}^{*}}} \\
\beta \leq q \leq \frac{\beta p_{\mathcal{D}_{\text {min }}^{*}}}{1-}
\end{gathered}
$$

This is a mixed-integer non-linear programming problem the solution of which is the optimal values of $p$ and $q$ as well as the maximum permissible SU transmit powers $P_{\max }^{(i)}$ (and hence, the corresponding maximum transmission rates $R_{\max }^{(i)}$ ) over each of the $N$ channels. Solving such a mixed-integer non-linear programming problem is NP hard. In what follows, we present an exhaustive study of the impact of different factors over the solution of the problem, and hence, the achievable CRN user rate. We use MATLAB for our simulations. We consider 4 PRNs distributed over a $500 \times 500$ square meter area each with 200 users using the $\{0.769$, $0.925,2.412,5.180\} \mathrm{GHz}$ channels with power masks of $2 \mathrm{nW}$ and channel bandwidth $B_{i}=20 \mathrm{MHz}$ for all channels. Other simulation parameters are $d_{o}=\{42,33,12$, 6) $\mathrm{cm}, P_{\mathrm{PU}}^{(i)}=1 \mathrm{~W}, G_{T}^{(i)}=G_{R}^{(i)}=1$ for all $i, n=4$, and $d_{c}$ $=50 \mathrm{~m}$ for $-80 \mathrm{~dB}$ receive sensitivity. A SU-TX picks its rate from $\{54,36,24,12,2\}$ Mbps with the power of the $54 \mathrm{Mbps}$ rate is $1 \mathrm{~W}$, and the corresponding power of other rates is computed using (1).

Impact of $\boldsymbol{p}_{D_{\text {min }}^{*}}$

The only variable in the above problem formulation is $p_{\mathcal{D}_{\text {min }}^{*}}$, which reflects the accuracy of the minimum distance between a secondary sender and a primary receiver. Figure 2 depicts the optimal $p$ and $q$ values and the CRN user rate versus the PRN activity factor for different $p_{\mathcal{D}_{\text {min }}^{*}}$ values for $\beta=5 \%$. As shown in Figure $2 \mathrm{a}$, the optimal probability of transmission over a clear spectral opportunity, $p$, depends significantly on the choice of $p_{\mathcal{D}_{\text {min }}^{*}}$ and tends to be the maximum possible value of $\beta /\left(1-p_{\mathcal{D}_{\min }^{*}}\right)$. However, the PRN activity factor does not impact $p$ as $p$ is the probability of using the highest possible power/rate conditioning on the lack of nearby PRN activities. On the other hand, $q$, the probability of SU transmission given PRN activities in the vicinity of the SU-TX, varies with both $p_{\mathcal{D}_{\text {min }}^{*}}$ and the PRN activity 


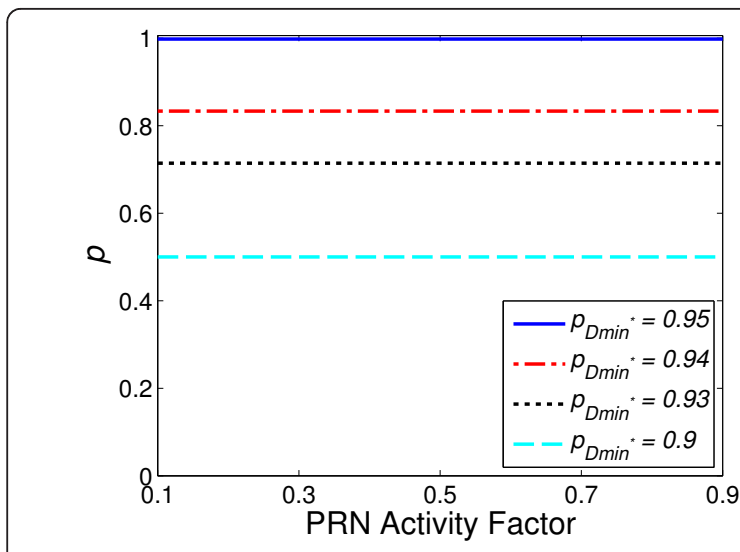

(a) Clear spectrum transmission probability.

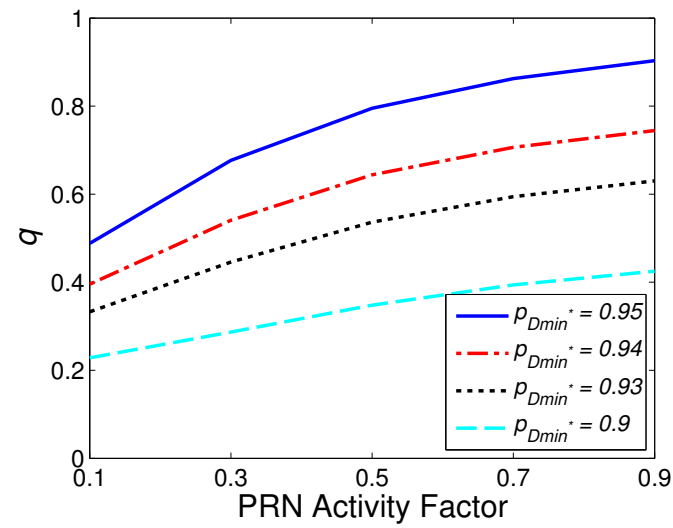

(b) Unclear spectrum transmission probability.

Figure 2 Optimal transmission probabilities for different PRN activity factors and $\boldsymbol{p}_{\mathcal{D}_{\text {min }}^{*}}$ a Clear spectrum transmission probability; b Unclear spectrum transmission probability.

factor as illustrated in Figure 2b. As the PRN activities increase, $q$ also increases to allow RAP-MAC to explore potentially missed opportunities more frequently to maximize the CRN user rate.

\section{Impact of the PRN Outage Constraint}

Next, we evaluate the impact of the maximum outage probability allowed by the PRNs, $\beta$. We solve (22) for $\beta$ equals to 1,5 , and $10 \%$. For the stringent outage constraint of $\beta=1 \%$, both $p$ and $q$ fall rapidly as $p_{\mathcal{D}_{\text {min }}^{*}}$ decreases as shown in Figure 3 . Recall that $p_{\mathcal{D}_{\text {min }}^{*}}$ represents how $\mathcal{D}_{\text {min }}^{*}$ is close to the distance at which outage occurs with probability equal to unity. Hence, as $p_{\mathcal{D}_{\text {min }}^{*}}$ decreases, RAP-MAC tends to be more conservative (i. e., lower $p$ and $q$ values) in order not to violate the PRN constraints. However, as $\beta$ increases, the impact of $p_{\mathcal{D}_{\text {min }}^{*}}$ on the optimal values of $p$ and $q$ is reduced. As shown in Figure 3, $p$ and $q$ fall slowly for $\beta=5$ and $10 \%$. Note that the PRN activity factor only impacts the value of $q$ (but not $p$ ) as explained earlier regardless of the value $\beta$. However, the impact of the PRN activity factor on $q$

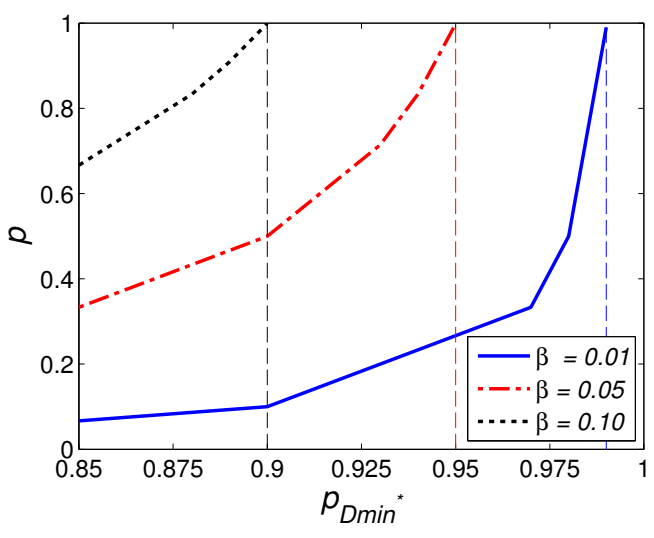

(a) Clear spectrum transmission probability.

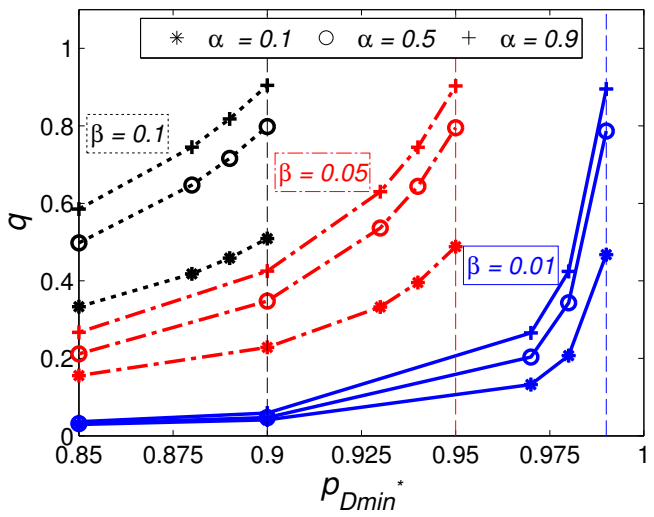

(b) Unclear spectrum transmission probability.

Figure 3 Impact of $\beta$ and $\boldsymbol{p}_{\mathcal{D}_{\min }^{*}}$ on the optimal transmission probabilities for different PRN activity factors min. a Clear spectrum transmission probability; b Unclear spectrum transmission probability.

increases with the relaxation of the PRN constraint $\beta$ as shown in Figure 3b.

\section{CRN User Rate}

Despite the strong dependencies of the optimal value of $p$ and $q$ on $p_{\mathcal{D}_{\text {min }}^{*}}$, Figure 4 a shows that $p_{\mathcal{D}_{\text {min }}^{*}}$ has a minimal impact on the maximum rate of CRN users. While the closer $p_{\mathcal{D}_{\text {min }}^{*}}$ to $1-\beta$ achieves the highest $\mathrm{CRN}$ rate, using smaller values for $p_{\mathcal{D}_{\text {min }}^{*}}$ achieves very close CRN rate. For example, the CRN rate using $=0.94$ is only 1 $2.8 \%$ (depending on the PRN activity factor) less than the rate when $p_{\mathcal{D}_{\text {min }}^{*}}=0.95$. Note that the $C R N$ rate deteriorates with the increase in the PRN activity. Meanwhile, using $p_{\mathcal{D}_{\text {min }}^{*}}=0.94$ instead of 0.95 changes $p$ from 0.833 to 0.714 , which allows a bigger probabilistic capacity margin for multiple SUs to share available opportunities. Similar results were obtained for other values of $\beta$. Figure $4 \mathrm{~b}$ depicts the loss in the CRN user rate versus the offset in $p_{\mathcal{D}_{\text {min }}^{*}}$ from its maximum value of $1-\beta$ for different values of $\beta$ and $\alpha$. The 


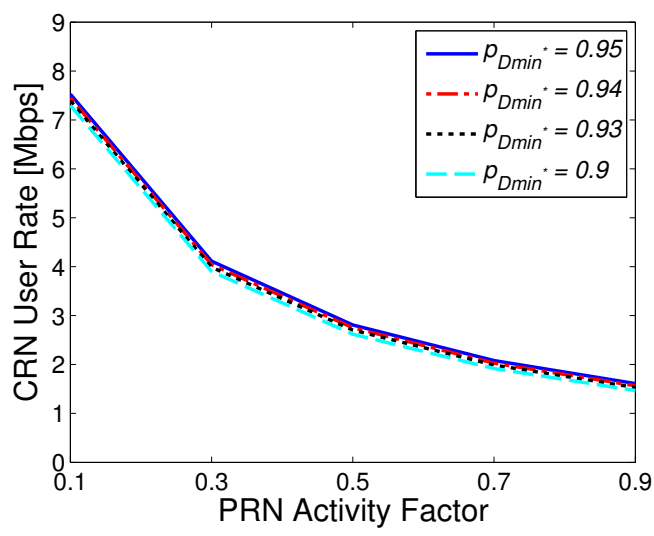

(a) CRN flow rate for $\beta=5 \%$.

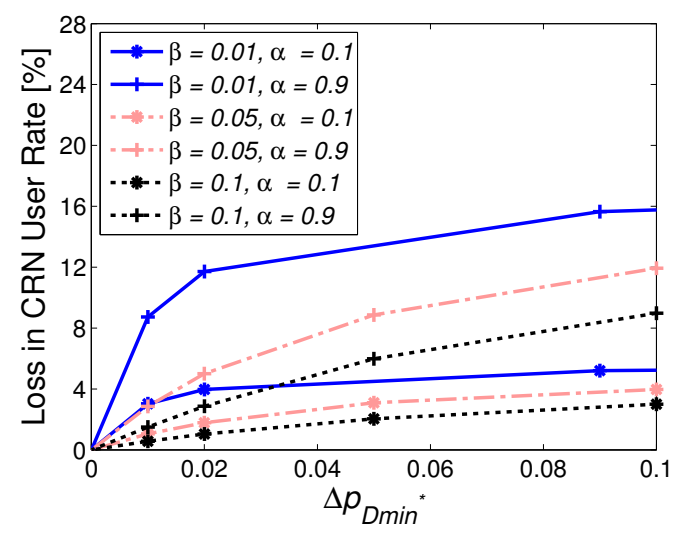

(b) Loss in CRN flow rate versus the offset in $p_{\mathcal{D}_{m i n}^{*}}^{*}$

Figure 4 The optimal CRN user rate and the impact of $\beta$ and $\boldsymbol{p}_{\mathcal{D}_{\text {min }}^{*} \text { a }}$ CRN flow rate for $\beta=5 \%$; b Loss in CRN flow rate versus the offset in $p_{\mathcal{D}_{\text {min }}^{*}}$

deterioration in the CRN user rate with $p_{\mathcal{D}_{\min }^{*}}$ increases as the PRN constraint $\beta$ gets tighter and the PRN activity factor $\alpha$ increases.

\section{RAP-MAC performance evaluation}

In this section, we evaluate the performance of the RAPMAC protocol. We develop an event-driven packet-level simulator. We consider 9 PRNs collocated with a CRN in a $500 \times 500$ square meter area. Each network has 200 nodes forming 100 sender-receiver pairs. The operating frequencies of the 9 PRNs are $\{0.769,0.789,0.809$, 2.412, 2.432, 2.462, 5.180, 5.200, 5.220\} GHz with respective activity factors of $\{0.1,0.5,0.9,0.1,0.5,0.9$, $0.1,0.5,0.9\}$. The bandwidth of each channel is 20 $\mathrm{MHz}$, and the power mask is $2 \mathrm{nW}$ for all PRNs. The PRN transmit power is $1 \mathrm{~W}$, and the transmit and receive antenna gains are equal to unity for all PRNs. We consider PRN maximum allowed outage probability values of 1,5 , and $10 \%$. The path loss exponent $n$ is set to be 4 . A secondary transmission can use a rate in the set $\{54,36,24,12,2\}$ Mbps. The corresponding set of transmission powers is calculated according to (1) with the transmission power of the $54 \mathrm{Mbps}$ rate is equal to $1 \mathrm{~W}$. We vary the arrival rate of all CRN users from 1 Mbps to 35 Mbps. For each arrival rate value, we generate 10 random node topologies. For each topology, we generate 3 traffic matrices. The reported results are the average of these 30 runs for each arrival rate value. The error bars represent the $95 \%$ confidence interval of the multiple runs. We use (22) to compute the optimal values of $p$ and $q$ for different values of $\beta$.

Our benchmark is a protocol that belongs to the family of hypothetically optimal spectrum access protocols which has a wide-sense capability and a greedy spectrum approach in the sense that a SU-TX exploits the best spectral opportunity at the maximum permissible power/rate. We use [16] to compute such maximum powers/rates. In order to insure fairness in comparison, we do not implement the capability of a secondary user to simultaneously transmit over multiple spectrum bands at a given time instant as in the protocol presented in [16]. We refer to such a modified protocol as OPT-MAC as it represents a wide range of spectrum access protocols that adopt greedy spectrum access mechanisms for transmission over available spectral opportunities (e.g., $[12,18,22])$. OPT-MAC spectrum access mechanism is carrier sensing based that uses message exchange over the common control channel to insure a single secondary user transmission per contention area. For each randomly generated topology and arrival process, we run both the RAP-MAC and OPTMAC protocols to guarantee fairness in comparison. Data packets are 1,500 bytes long for both protocols. Control packets of both protocols are 40 bytes transmitted at 12 Mbps rate over the common control channel. Spectrum sensing and transceiver turnaround times are 9 and $5 \mu \mathrm{s}$, respectively. The exponential backoff window is bounded by $(16,1,024)$ slots of $2-\mu$ s duration. Our performance metrics are the CRN average goodput, Jain's index as a measure of the fairness in CRN goodput distribution [24], and the outage probability of the PRNs defined as the probability of PRNs transmission failure due to CRN activities.

\section{CRN Goodput}

Figure 5a depicts the average goodput of CRN users using both the RAP-MAC and OPT-MAC for $\beta$ equals to $5 \%$. RAP-MAC achieves significantly higher goodput compared to OPT-MAC. The RAP-MAC gain in the CRN user goodput varies between 65 and 119.5\% depending on the CRN traffic demand. RAP-MAC significant gain in goodput is attributed to the fact that: $(i)$ 


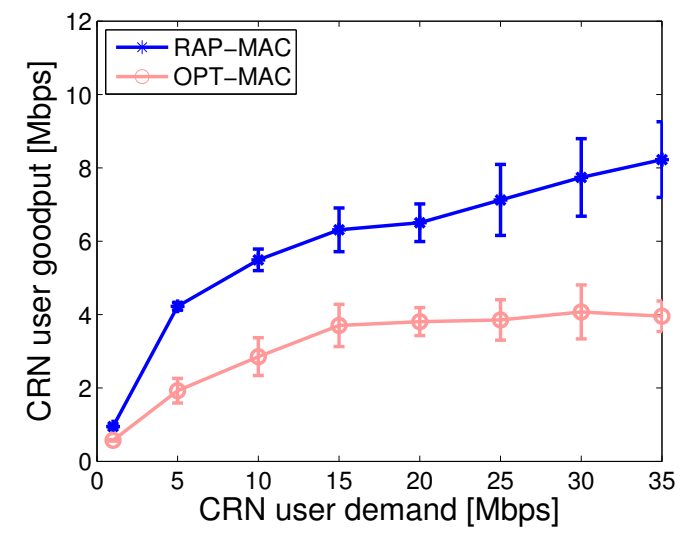

(a) CRN user goodput for $\beta=5 \%$.

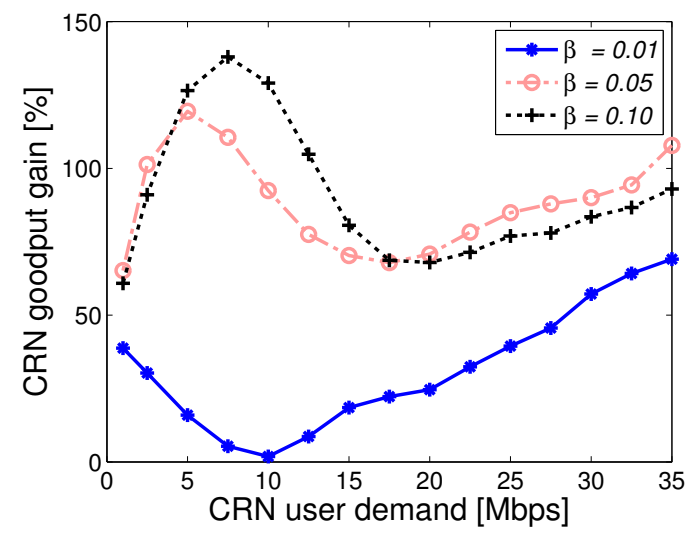

(b) Gain in CRN user goodput.

Figure 5 RAP-MAC achieves significant goodput gain for different $\boldsymbol{\beta}$ values. a CRN user goodput for $\beta=5 \%$; b Gain in CRN user goodput.

RAP-MAC probabilistically (with probability $q$ ) explores the spectral opportunities that OPT-MAC does not explore at all when the interference measurements imply unclear opportunities; and (ii) RAP-MAC is less susceptible to transmission failures (compared to OPTMAC) due to its probabilistic non-greedy policy for the clear spectrum situations which allows multiple flows to simultaneously use a given spectrum at highly reliable lower transmission rates. Consequently, the RAP-MAC gain depends on the value of $\beta$ which affects the optimal values of $p$ and $q$ as explained in Section 4. Figure 5b depicts the RAP-MAC gain for different $\beta$ values. As $\beta$ increases, the gain in the CRN goodput increases up to $138 \%$ as the case with $\beta$ equals to $10 \%$. This is due to the fact that the value of $q$ obtained using (22) is 0.5 , 0.41 , and 0.15 for $\beta$ equals to 10,5 , and $1 \%$, respectively. Furthermore, Figure $5 \mathrm{~b}$ shows that the gain peaks at low CRN traffic demands then decrease before it linearly increases with the traffic demands at $\beta$ equals to 5 and $\% 10$. For instance, maximum gain of 119.5 and $138 \%$ is achieved at $5 \mathrm{Mbps}$ and $7.5 \mathrm{Mbps}$ for $\beta$ equals to 5 and $10 \%$, respectively. However, the RAP-MAC goodput gain decreases before increasing again for the more stringent outage constraint of $\beta$ equals to $1 \%$.

As we mentioned earlier, the superior goodput performance of RAP-MAC is due to the bigger gap between its transmission attempts and transmission blockage (due to either PRN or CRN activities) compared to OPT-MAC as shown in Figure 6 a for $\beta$ equals to $5 \%$. As $\beta$ increases, the gap between the blocked and attempted transmissions increases. Regardless of the value of $\beta$, the number of transmission attempts of RAP-MAC (the solid stared line) is only slightly higher than that of OPT-MAC (the solid circled line). However, OPT-MAC transmissions are susceptible to more blockages as it does not account for the activities of hidden PRN or CRN nodes (the dashed lines). Recall that OPT-MAC allows a CRN sender either to transmit at the highest possible power/rate or to not transmit at all. Meanwhile, RAP-MAC has a secondary flow probabilistically adapt its power/rate based on the interference scenario. Figure $6 \mathrm{~b}$ depicts the distribution of the rates used by RAP-MAC under low and high CRN traffic demands. At high CRN demand, RAP-MAC tends to have the CRN flows using the minimum rate more often to allow multiple CRN flows to simultaneously share spectral opportunities. As the CRN demands decrease, RAP-MAC tends to use higher rates (might be through switching to a different spectrum) in order to minimize the unutilized capacity of available spectral opportunities. As shown in Figure 6b, RAP-MAC uses $R_{\min }$ for only $7.3 \%$ of the time at $1 \mathrm{Mbps}$ CRN demand compared to $22 \%$ at $35 \mathrm{Mbps}$ CRN demand. Figure 6 explains how RAP-MAC spectrum access decisions result in higher goodput as was illustrated in Figure 5.

\section{CRN Fairness}

RAP-MAC does not have an explicit mechanism for inter-flow coordination. However, it adopts a probabilistic non-greedy transmission approach that prevents a single CRN flow from exclusively capturing an available spectral opportunity. This results in RAP-MAC significantly outperforming OPT-MAC in terms of the fairness characteristics as shown in Figure 7. Figure 7a depicts Jain's Fairness Index JFI $=\frac{\left(\sum_{i} T(i)\right)^{2}}{L \sum_{i} T(i)^{2}}$, where $T(i)$ is the goodput of the $i$ th flow and $L$ is the number of CRN flows [24]. At low CRN demands, JFI of RAP-MAC approaches its optimal value of unity, implying that all flows are getting approximately equal goodput shares. As the traffic demands increase, JFI of RAP-MAC decreases, but it is always much higher than JFI of OPT-MAC. The poor fairness performance of OPTMAC is attributed to its greedy transmission strategy 


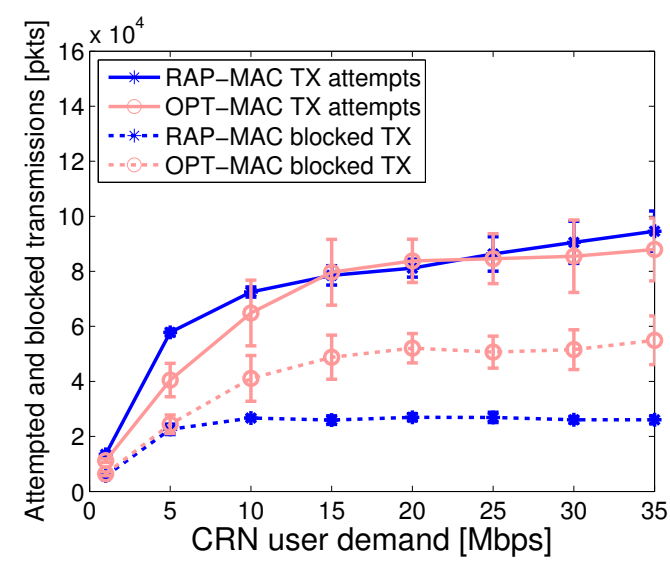

(a) Attempted and blocked transmission attempts for $\beta=5 \%$.

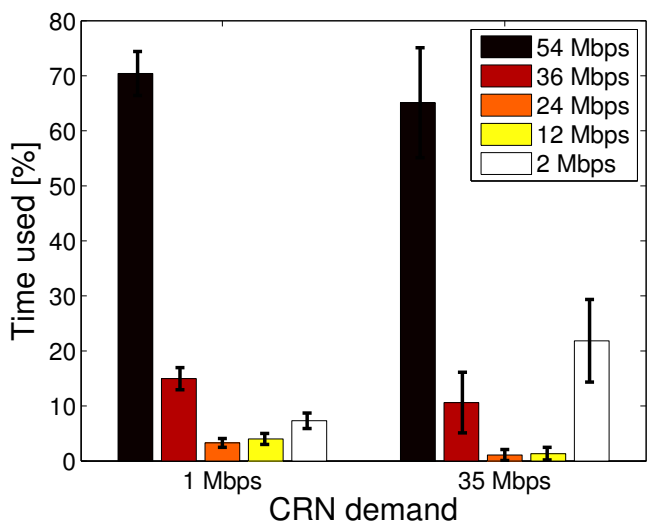

(b) Distribution of rates used by RAP-MAC for $\beta=5 \%$.

Figure 6 RAP-MAC spectrum access decisions lead to fewer blocked transmission attempts. a Attempted and blocked transmission attempts for $\beta=5 \%$; b Distribution of rates used by RAP-MAC for $\beta=5 \%$.

that can allow some flows to exclusively capture spectral opportunities and thereby starving other flows in ad hoc networks. Figure $7 \mathrm{~b}$ illustrates the percentage of flows receiving less than $10 \%$ of the average CRN goodput. It can be seen that OPT-MAC allows only $53 \%$ of the flows to capture the spectral opportunities starving the remaining $47 \%$ of the flows. Meanwhile, less than 1 and $2 \%$ of the flows are underserved using RAP-MAC depending on the value of $\beta$.

\section{Channel Utilization}

OPT-MAC assumes CRN nodes with wideband spectrum sensing capability aiming at closely tracking spectral opportunities. Meanwhile, RAP-MAC has the CRN flows randomly picking their channels. Despite the difference in the spectrum sensing scheme, Figure 8 shows that both RAP-AMC and OPT-MAC tend to utilize the channels licensed to PRNs with the lowest activity

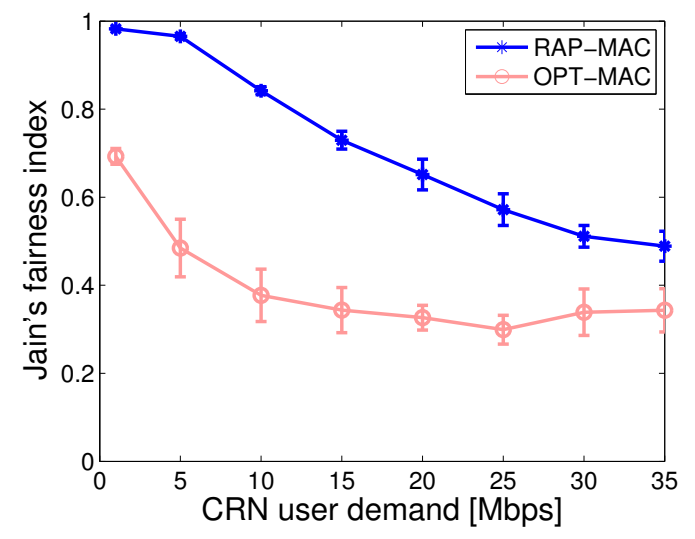

(a) Jain's fairness index.

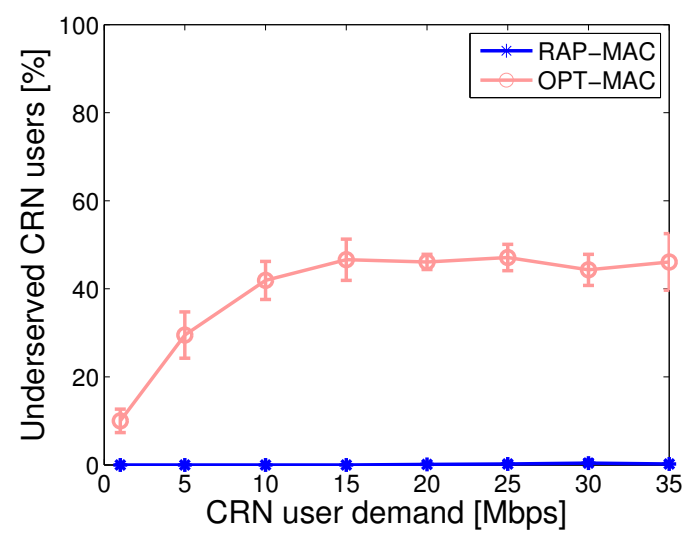

(b) Percentage of underserved CRN flows.

Figure 7 RAP-MAC fairly distributes spectral opportunities among different users without explicit inter-flow coordination. a Jain's fairness index; b Percentage of underserved CRN flows.

factors of 0.1 for most of the time, namely channels 1 , 4 , and 7 illustrated by the dark blue, light blue, and orange bars, respectively. At low CRN traffic demands, both RAP-MAC and OPT-MAC do not frequently utilize the rest of the channels with activity factors of 0.5 and 0.9 as illustrated in Figure 8a. However, as the CRN traffic demand increases (Figure 8b), RAP-MAC probabilistic access scheme allows the CRN flows to explore the heavily utilized channels more than OPT-MAC rather than having the excess demand utilizing channels 1,4 , and 7. However, RAP-MAC does not degrade the outage performance of highly active PRNs because of RAP-MAC probabilistic access as discussed next. Such distribution of transmissions over more channels decreases the amount of blocked and failed CRN transmission attempts.

\section{PRN Outage}

Finally, we investigate the outage performance of the primary licensed networks. Figure 9 depicts the outage 


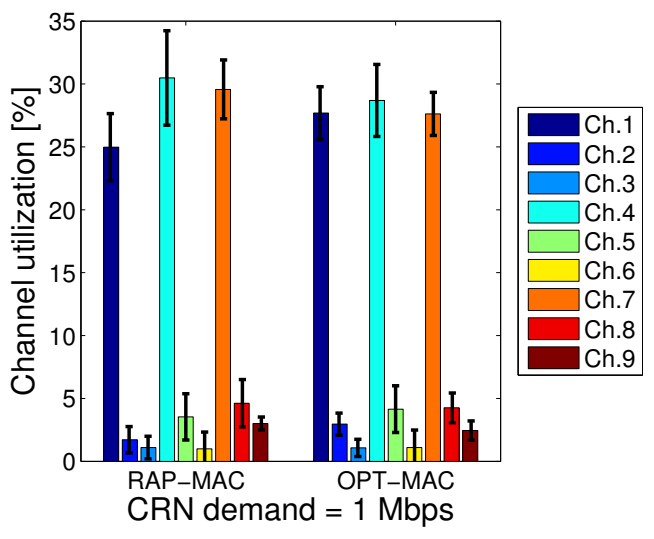

(a) Low CRN demand.

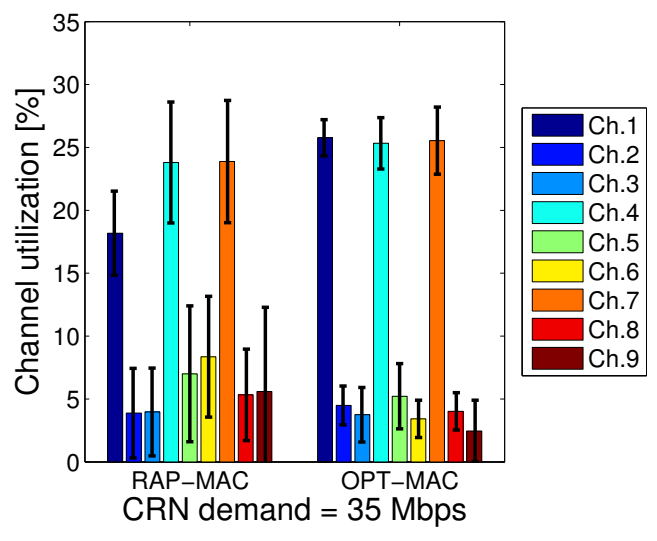

(b) High CRN demand.

Figure 8 The distribution of channel utilization for $\beta$ equals to 5\%. a Low CRN demand; b High CRN demand.

probability of PRNs using channel 1,4 , and 7 for both RAP-MAC and OPT-MAC for $\beta$ equals to $5 \%$. These channels are the highest channels exploited by the CRN. Recall that the frequency of channel 1 is lower than that of channel 4 that is lower than the frequency of channel 7. Due to the better propagation characteristics at lower frequencies, both the RAP-MAC and the OPT-MAC protocols favor (i.e., initiate more transmission attempts) channel 1 more than channel 4 more than channel 7 . Consequently, the outage probability of the PRN using channel 1 is the highest and that of channel 7 is the lowest. For other PRNs with higher activity factors, the outage probability is insignificant for both protocols (below 0.001 and hence are not shown in Figure 9). For all channels, the PRN outage probability due to RAPMAC (represented by solid lines) is higher than that due to OPT-MAC (represented by dotted lines) because of the RAP-MAC probabilistic transmission policy. However, the outage due to RAP-MAC is always below the PRN specified bound irrespective of the value of $\beta$ and the CRN traffic demand.

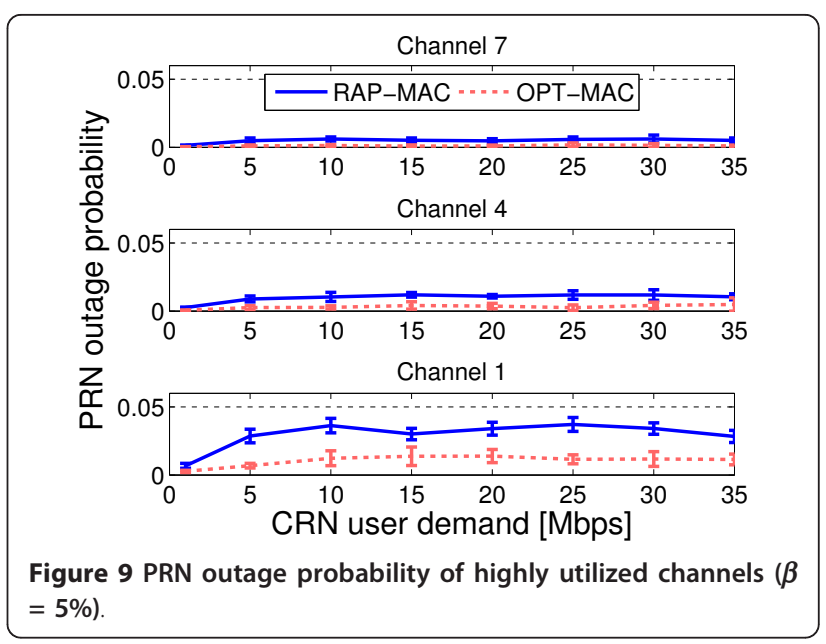

\section{6. related work}

The literature of spectrum management in wireless cognitive networks is affluent and covers various aspects such as spectrum sensing, spectrum access, and spectrum sharing. For an in-depth discussion of various schemes, please refer to [3-5]. Here, we briefly discuss the closely related literature.

\section{Opportunistic Spectrum Sensing}

The problem of finding which frequency bands to sense and probe before transmission has been widely addressed in the context of both multi-channel and cognitive radio networks (see [5] and references therein). Recently, the focus of the related literature was to relax the assumptions/requirements of the sensing module of a cognitive radio. For instance, adopting only a subset of the available frequency bands to probe has been proposed in $[17,18]$ based on distributed learning techniques. In [25], the authors compute the network capacity when only a subset of the available frequency bands is to be used due to transceiver hardware constraints. Both adjacent and random channel assignment models were considered. Alternatively, relaxing the amount of information needed to assess the existence of spectral opportunities was addressed in [6-8]. Compressed sensing [6] techniques and randomized sensing $[7,26]$ and sampling [8] were proposed. However, all of the aforementioned sensing techniques lead to inaccurate decisions in hidden or exposed primary sender scenarios. Consequently, CRN performance optimization while overlooking such inherent inaccuracy does not lead to optimal performance in all scenarios. One way to address the spectrum sensing limitations is to exploit the bidirectional communication nature in some primary networks $[27,28]$. By monitoring the reverse traffic originated from primary receivers, secondary senders can infer the existence or the absence of nearby primary receivers. 
However, such schemes are not applicable to all primary networks. Furthermore, such schemes still cannot provide information about the actual interference at the primary receivers. Hence, they cannot help the secondary users determining the appropriate transmission parameters.

\section{Opportunistic Spectrum Access and Sharing}

The spectrum access problem is to determine the resources to be used for an upcoming transmission. Such a resource allocation decision includes both the identity of the spectrum to be used along with a transmission scheme to be used (defined in terms of the transmission power and the modulation rate) and the time instance such a spectrum is available. On the other hand, the spectrum sharing problem considers multiuser scenarios and jointly allocates the available resources among different secondary flows. Due to the close relationship between the two problems, they are generally jointly addressed. Several spectrum access and sharing schemes have been proposed for CRNs with the general objective of maximizing the CRN goodput without violating the interference (and consequently, outage) constraints of the primary licensed networks [3,4,9-18]. One way to classify spectrum access and sharing schemes is based on how the resource allocation decisions are made as follows:

\section{Centralized Spectrum Access/Sharing}

Such schemes rely on a single centralized entity that collects the spectrum measurements from different nodes and makes the spectrum access decisions and resource allocation decisions for different transmissions. In [9], a spectrum server is utilized to find the optimal schedule that maximizes the average sum rate subject to a minimum average rate constraint for cognitive links using a graph-theoretic approach. The resulting schedules are a collection of transmission modes (sets of active links) that are time shared in a fashion that is reminiscent of spatial reuse patterns in cellular networks. In [10], a joint admission control and resource scheduling policy is proposed based on Laya-punov optimization techniques. Alternatively, [11] adopts a gametheoretic approach to find the optimal channel assignments and transmission powers/rates. The authors of [11] consider the IEEE 802.22 network model in which a set of base stations are responsible for spectrum access and management and analyze the performance of both cooperative and noncooperative schemes.

\section{Distributed Spectrum Access/Sharing}

In a more related context, distributed spectrum management schemes have been proposed for cognitive ad hoc network [12-18]. CRN users individually or jointly decide their channel allocations and transmission powers and rates without a central coordinator. For instance, [12] adopts a decision-theoretic approach and presents an analytical framework that integrates the design of spectrum access protocols at the MAC layer, the spectrum sensing at the physical layer and the traffic statistics determined by the application layer of the licensed networks. In [13], different sensing-based opportunistic access schemes were proposed via modeling the primary users as $\mathrm{M} / \mathrm{G} / 1$ queues and introducing the collision probability and the overlapping time as the metrics for primary user performance. Meanwhile, [14] presents a price-based spectrum management framework for cognitive radio networks. The framework models the CRN problem as a noncooperative game and uses a price-based iterative water-filling algorithm to reach Nash equilibrium. Alternatively, [15] presents a novel cooperative game-theoretic paradigm that allows secondary users to freely optimize the channel utilization for transmitting the primary network data along with their own data. Learning techniques have also been employed to find the optimal resource allocation (time, spectrum, power, and rate) that maximizes the goodput the CRN $[17,18]$. In [17], two distributed cooperative learning and allocation schemes were proposed: one that assumes minimal prior knowledge of secondary user information and the other does not assume such information. The objective of both schemes is to minimize the total regret in distributed learning (or equivalently maximize the CRN goodput). Similarly, [18] utilizes adaptive learning for spectral probing that is integrated with the resource allocation to maximize the CRN goodput. The authors of [16] propose a CSMA/ CA-based MAC that does not rely on the interaction with the licensed network. Instead, resource allocation decisions are based on the statistics of the interference over different channels.

In contrast to all of the aforementioned distributed schemes, our RAP approach does not imply any interflow coordination mechanism. The proposed probabilistic and non-greedy access mechanism allows competing secondary flows to efficiently and fairly share the available spectral opportunities without relying on the common control channel for inter-flow communication. Hence, the common control channel is no longer the bottleneck of the CRN nor the single point of failure of the system.

\section{Conclusions}

In this paper, we have presented a framework for opportunistic spectrum management. Unlike prior work, we have adopted a probabilistic and non-greedy approach to counter the limitations of cognitive radio networks such as the inability to base the spectrum management decisions on the interference at primary receivers and the increased complexity of accurate high-speed 
wideband sensors. We have analytically formulated the constrained cognitive network achievable goodput as a mixed-integer non-linear programming problem to find the optimal parameter values that maximizes the achievable goodput. With optimized parameter values, the proposed RAP-MAC achieves more than $2 x$ improvement in the goodput performance of the cognitive users while satisfying the primary network outage constraints in ad hoc networks compared to greedy spectrum management schemes. Furthermore, RAP-MAC has an outstanding fairness performance, without using explicit inter-flow coordination, due to its non-greedy transmission policy.

\section{Endnotes}

${ }^{\text {a }}$ Recent works exploited the bidirectional nature of some primary networks to enable SUs to infer the existence or the absence of a neighboring primary receiver $[27,28]$. However, such schemes do not provide a way to measure the cumulative interference at the primary receiver. A more detailed discussion of the related work is presented in Section 6. ${ }^{\mathbf{b}} \mathrm{A}$ PRN can be licensed to use multiple contiguous or non-contiguous channels. However, our generalized assumption of different PRN per channel can be easily extended to capture such situations by dividing such a multi-band PRN into multiple virtual PRNs. ${ }^{c}$ We do not incorporate the ramp up from $R_{1}$ to $R_{\max -1}$. While such assumption slightly impacts the achievable rate of a SU, it does not affect our optimization problem as the outage constraints depend only on the maximum used rate. ${ }^{\mathbf{d}}$ In multiuser scenarios, RAP-MAC uses lower powers/rates. The interference caused by multiple weak sources has negligible impact (almost as AWGN noise) on ongoing transmissions compared to the interference from a single high power/rate source [20,21]. Hence, (20) also covers the multiuser case.

\section{Competing interests}

The authors declare that they have no competing interests.

Received: 25 February 2011 Accepted: 28 November 2011 Published: 28 November 2011

\section{References}

1. FCC, ET Docket No 03-222 Notice of Proposed Rule Making and Order (Federal Communications Comission (FCC), 2003)

2. J Mitola III, Cognitive Radio. An Integrated Agent Architecture for Software Defined Radio. Ph.D. dissertation (KTH Royal Institute of Technology, 2000)

3. IF Akyildiz, W-Y Lee, KR Chowdhury, Crahns: cognitive radio ad hoc networks. Ad Hoc Netw. Elsevier. 7(5), 810-836 (2009). doi:10.1016/j. adhoc.2009.01.001

4. HB Salameh, M Krunz, Channel access protocols for multihop opportunistic networks: challenges and recent developments. IEEE Netw. 23(4), 14-19 (2009)

5. T Yucek, H Arslan, A survey of spectrum sensing algorithms for cognitive radio applications. IEEE Commun Surv Tutor.11(1), 116-130. First Quarter (2009)
6. Z Tian, G Giannakis, Compressed sensing for wideband cognitive radios. in Proceedings of IEEE ICASSP. Honolulu (2007)

7. H Liu, B Krishnamachari, Randomized strategies for multi-user multi-channel opportunity sensing. in Proceedings of IEEE CCNC Cognitive Radio Networks Workshop. Las Vegas (2008)

8. Z Liang, W Liu, P Zhou, F Gao, Randomized multi-user strategy for spectrum sharing in opportunistic spectrum access network. in Proceedings of IEEE ICC Workshops. Beijing (2008)

9. C Raman, RD Yates, NB Mandayam, Scheduling variable rate links via a spectrum server. in Proceedings of the IEEE DYSPAN 2005. Baltimore (2005)

10. M Lotfinezhad, B Liang, ES Sousa, Optimal control of constrained cognitive radio networks with dynamic population size. in Proceedings of the IEEE INFOCOM 2010. San Diego (2010)

11. G Hosseinabadi, MH Manshaei, J-P Hubaux, Spectrum Sharing Games of Infrastructure-based Cognitive Radio Networks Technical Report 2008 http:// infoscience.epfl.ch/record/128112?!n=en

12. Q Zhao, L Tong, A Swami, Y Chen, Decentralized cognitive MAC for opportunistic spectrum access in ad hoc networks: a POMPD framework. IEEE J Sel Areas Commun. 25(3), 589-600 (2007)

13. S Huang, $X$ Liu, Z Ding, Opportunistic spectrum access in cognitive radio networks. in Proceedings of the IEEE INFOCOM 2008. (Phoenix) (2008)

14. F Wang, M Krunz, S Cui, Price-based spectrum management in cognitive radio networks. IEEE J Sel Top Signal Process. 2(1), 74-87 (2008)

15. H Xu, B Li, Efficient resource allocation with flexible channel cooperation in OFDMA cognitive radio networks. in Proceedings of the IEEE INFOCOM 2010. San Diego (2010)

16. HB Salameh, M Krunz, O Younis, MAC protocol for opportunistic cognitive radio networks with soft guarantees. IEEE Trans Mobile Comput. 8(10), 1339-1352 (2009)

17. A Anandkumar, N Michael, A Tang, Opportunistic spectrum access with multiple users: learning under competition. in Proceedings of IEEE INFOCOM 2010. San Deigo (2010)

18. P Chaporkar, A Proutiere, H Asnani, Learning to optimally exploit multichannel diversity in wireless systems. in Proceedings of the IEEE INFOCOM 2010. San Diego (2010)

19. D Cabric, RW Brodersen, Physical layer design issues unique to cognitive radio systems. in Proceedings of IEEE PIMRC. Berlin (2005)

20. T Rappaport, Wireless Communications, Principles \& Practice (Prentice Hall, Upper Saddle River, 1996)

21. A Khattab, An experimental case for SIMO random access in multi-antenna multi-hop wireless networks. in Proceedings of the IEEE INFOCOM 2010. San Diego (2010)

22. S Huang, X Liu, Z Ding, Optimal transmission strategies for dynamic spectrum access in cognitive radio networks. IEEE Trans Mobile Comput. 8(12), 1636-1648 (2009)

23. TC Clancy, Achievable capacity under the interference temperature model. in Proceedings of IEEE INFOCOM. Anchorage (2007)

24. R Jain, The Art of Computer Systems Performance Analysis (Wiley, New York, 1991)

25. V Bhandari, NH Vaidya, Capacity of multi-channel wireless networks with random $(c, f)$ assignment. in Proceedings of the ACM Mobihoc 2007. Montreal (2007)

26. Bl Ahmad, A Tarczynski, Reliable wideband multichannel spectrum sensing using randomized sampling schemes. Signal Process. 90(7), 2232-2242 (2010). doi:10.1016/j.sigpro.2010.02.006

27. FE Lapiccirella, Z Ding, $X$ Liu, Cognitive spectrum access control based on intrinsic primary ARQ information. in Proceedings of IEEE ICC 2010. Cape Town (2010)

28. C-H Lee, M Haenggi, Delay analysis of spatio-temporal channel access for cognitive networks. in Proceedings of IEEE ICC 2011. Kyoto (2011)

doi:10.1186/1687-1499-2011-188

Cite this article as: Khattab et al.: Probabilistic framework for opportunistic spectrum management in cognitive ad hoc networks. EURASIP Journal on Wireless Communications and Networking 2011 2011:188. 